\title{
Prostaglandin E Receptor EP4 Antagonist Suppresses Lipopolysaccharide-Induced Osteoclast Formation and Inflammatory Bone Loss
}

\author{
Chiho Matsumoto, ${ }^{a}$ Morichika Takita, ${ }^{a}$ Masaki Inada, ${ }^{a}$ Takayuki Maruyama, ${ }^{b}$ \\ and Chisato Miyaura*,a \\ ${ }^{a}$ Department of Biotechnology and Life Science, Tokyo University of Agriculture and Technology, 2-24-16 Nakamachi, Koganei, \\ Tokyo 184-8588, Japan and ${ }^{b}$ Discovery Research Laboratories I, Minase Research Institute, Ono Pharmaceutical Co., Ltd., \\ 3-1-1 Sakurai, Shimamoto-cho, Mishima-gun, Osaka, Japan
}

(Received November 29, 2006; Accepted December 12, 2006; Published online December 19, 2006)

Prostaglandin $\mathrm{E}_{2}\left(\mathrm{PGE}_{2}\right)$ is mainly produced by osteoblasts in bone tissue, and acts as a potent stimulator of bone resorption. In osteoblasts, $\mathrm{PGE}_{2}$ production was greatly stimulated by lipopolysaccharide (LPS) following the expression of cyclooxygenase (COX)-2 and membrane-bound PGE synthase (mPGES)-1 mRNA. In the coculture of mouse bone marrow cells and osteoblasts, LPS induced PGE production and osteoclast formation, and EP4 antagonist completely suppressed osteoclast formation, indicating that the $\mathrm{PGE}_{2}$-mediated EP4 signal is essential for LPS-induced osteoclast formation. Inflammatory bone diseases including periodontitis are known to be accompanied by bone loss with increased osteoclastogenesis. To examine the role of EP4-mediated $\mathrm{PGE}_{2}$ action in periodontitis, we examined the effects of EP4 antagonist on LPS-induced bone resorption using mouse alveolar bone. In an organ culture of alveolar bone, LPS-induced bone resorbing activity and EP4 antagonist suppressed this LPS-induced bone resorption. In an experimental model of periodontitis, LPS was injected into the lower gingiva, and the bone mineral density of alveolar bone was measured. LPSinduced the loss of alveolar bone, which was recovered by the treatment with EP4 antagonist in vivo. Therefore, EP4 antagonist is a possible candidate for the therapy of inflammatory bone disease including periodontitis.

Key words — prostaglandin E, EP4 receptor, bone resorption, inflammation, periodontitis

\footnotetext{
*To whom correspondence should be addressed: Department of Biotechnology and Life Science, Tokyo University of Agriculture and Technology, 2-24-16 Nakamachi, Koganei, Tokyo 184-8588, Japan. Tel.: +81-42-388-7390; Fax: +81-42-3887390; E-mail: miyaura@cc.tuat.ac.jp
}

\section{INTRODUCTION}

Bone turnover is regulated by osteoclastic bone resorption and new bone formation mediated by osteoblasts. Recent studies have established that most bone-resorbing factors act on osteoblasts to induce receptor activator of NF- $\kappa \mathrm{B}$ ligand (RANKL), a key molecule in osteoclast differentiation. ${ }^{1-3}$ ) Prostaglandin $\mathrm{E}_{2}\left(\mathrm{PGE}_{2}\right)$ is produced mainly by osteoblasts in bone and stimulates bone resorption, especially during inflammation. $\mathrm{PGE}_{2}$ synthesis is regulated in three metabolic steps: the release of arachidonic acid from membranous phospholipids by phospholipase A2 (PLA2), the conversion of arachidonic acid to $\mathrm{PGH}_{2}$ by cyclooxygenase (COX), and the synthesis of $\mathrm{PGE}_{2}$ by PGE synthase (PGES). ${ }^{4-6)}$ We have reported that the role of cytosolic $\mathrm{PLA}_{2}\left(\mathrm{cPLA}_{2}\right)$ expression in osteoblasts is to release arachidonic acid following $\mathrm{PGE}_{2}$ production. ${ }^{7,8)}$ Two isoforms of the COX enzyme, COX1 and COX-2, have been identified, and previous studies have shown that COX-1 is a constitutive enzyme and COX-2 is an enzyme induced by various inflammatory stimuli. In osteoblasts, the expression of COX-2, but not COX-1, is markedly induced by interleukin-1 (IL-1). The terminal step in $\mathrm{PGE}_{2}$ synthesis is catalyzed by PGES for the conversion of $\mathrm{PGH}_{2}$ to $\mathrm{PGE}_{2}$. Recent studies have identified three forms of PGES: cytosolic PGES (cPGES), membrane-bound PGES (mPGES)-1, and mPGES$2 .^{6,9-11)}$ It is known that mPGES-1 is markedly induced by inflammatory stimuli, and functionally coupled with COX-2 for the accumulation of $\mathrm{PGE}_{2}$ during inflammation.

There are four subtypes of PGE receptors, designated EP1, EP2, EP3, and EP4, that are encoded by different genes and expressed differently 
in each tissue. ${ }^{12)}$ The intracellular signaling differs among the receptor subtypes: EP1 is coupled to calcium mobilization, EP3 inhibits adenylate cyclase, whereas both EP2 and EP4 stimulate adenylate cyclase in various types of cells. Using knockout mice of respective EPs and specific EP agonists, we reported that $\mathrm{PGE}_{2}$ stimulates bone resorption mainly by EP4 signal. ${ }^{13,14)}$ EP4 agonist greatly stimulated the expression of RANKL in osteoblasts and induced osteoclast formation in mouse bone marrow cultures. ${ }^{13)}$ Therefore, it may be possible that blocking of the EP4 signal using EP4 antagonist prevents bone loss in inflammatory bone diseases.

Lipopolysaccharide (LPS) is a known pathogen leading to periodontitis, an infectious disease of mixed gram-negative bacteria that is associated with bone resorption. Toll-like receptors (TLRs) are known to play a critical role in innate immune responses in mammals, and 11 members of the TLR family (TLR1-TLR11) have been identified. ${ }^{15)}$ The TLRs are thought to be receptors for the recognition of various ligands, including bacterial components and nucleic acids that are involved in host defense mechanisms against pathogens. ${ }^{15)}$ Previous studies have shown that TLR4 is the most essential LPS receptor for signal transduction. ${ }^{16)}$ Not only immune systems, but also infectious diseases such as periodontitis, are thought to be involved in LPS-induced inflammation and the ensuing tissue destruction.

In the present study, we examined the effects of EP4 antagonist on LPS-induced osteoclast formation and destruction of alveolar bone, and suggest that blockage of the EP4 signal is a new therapeutic approach for inflammatory bone disease including periodontitis.

\section{MATERIALS AND METHODS}

Animals and Reagents — Newborn and 6-weekold mice of $d d y$ strains were obtained from Japan SLC Inc. (Shizuoka, Japan). All procedures were performed in accordance with the institutional guidelines for animal research. LPS (Escherichia coli 055:B5) was purchased from Difco Laboratories (Detroit, MI, U.S.A.). $\mathrm{PGE}_{2}$ was obtained from Sigma (St. Louis, MO, U.S.A.). EP4 antagonist (ONO-AE3-208) was prepared in Ono Pharmaceutical Co., Ltd. Osaka, Japan.

Culture of Primary Mouse Osteoblastic Cells _ Primary osteoblastic cells were isolated from newborn mouse calvariae after five routine sequential digestions with $0.1 \%$ collagenase and $0.2 \%$ dispase. ${ }^{7)}$ Osteoblastic cells collected from fractions 2-5 were combined and cultured in $\alpha$-modified minimum essential medium ( $\alpha \mathrm{MEM})$, supplemented with $10 \%$ fetal calf serum (FCS) at $37^{\circ} \mathrm{C}$ under $5 \%$ $\mathrm{CO}_{2}$ in air.

Measurement of $\mathrm{PGE}_{\mathbf{2}}$ Content — The concentrations of $\mathrm{PGE}_{2}$ in the cultured medium were determined using an enzyme immunoassay (EIA) (Amersham, Aylesbury, U.K.). The antibody had the following cross-reactivity when calculated using the bound/free ratio: $\mathrm{PGE}_{2}, 100 \%$; $\mathrm{PGE}_{1}, 7.0 \%$; 6keto-PGF $1 \alpha, 5.4 \%$; $\mathrm{PGF}_{2} \alpha, 4.3 \%$; and $\mathrm{PGD}_{2}, 1.0 \%$. Osteoclast Formation in Coculture of Mouse Bone Marrow Cells and Osteoblasts - Bone marrow cells $\left(3 \times 10^{6}\right.$ cells $)$ were isolated from 6week-old mice and cocultured with the primary osteoblastic cells $\left(1 \times 10^{4}\right.$ cells $)$ in $1 \mathrm{~mL}$ of $\alpha \mathrm{MEM}$ containing 10\% FCS. After being cultured for 7 days, the cells were stained for tartrate-resistant acid phosphatase (TRAP), and TRAP-positive multinucleated cells were counted as osteoclasts.

reverse transcriptase (RT)-PCR Analysis Total RNA was extracted from cultured cells using the acid guanidium-phenol-chloroform method, and cDNA was synthesized from $5 \mu \mathrm{g}$ of total RNA by reverse transcriptase using PCR. ${ }^{17)}$ The primers in the PCR for the mouse COX-1, COX-2, mPGES1, cPGES, and glyceraldehyde-3-phosphate dehydrogenase (GAPDH) genes were used as reported previously. ${ }^{17)}$ The PCR product was run on a $1.5 \%$ agarose gel and stained with ethidium bromide.

Bone-resorbing Activity in Organ Culture of Mandibular Alveolar Bone —_ Mandibular alveolar bone was collected from mouse lower gingivae under a microscope, and cultured for $24 \mathrm{hr}$ in BGJb containing $1 \mathrm{mg} / \mathrm{ml}$ of bovine serum albumin. After $24 \mathrm{hr}$, alveolar bone was transferred into new medium with or without LPS, and cultured for 5 days. The concentration of calcium in the conditioned medium was measured by the ocresolphthalein complexon (OCPC) method. ${ }^{14)}$ The bone-resorbing activity was expressed as an increase in medium calcium, as shown in previous studies. ${ }^{14)}$

Treatment with LPS in Mice-As a model for experimental periodontitis, LPS $(25 \mu \mathrm{g})$ was injected into six-week-old mouse lower gingivae on days 0,2 , and 4 . As the control, PBS was injected into the lower gingivae. Mandibular alveolar bone was collected 7 days after the first injection. 
Radiographic Analysis — The bone mineral density (BMD) of mandibular alveolar bone was measured by dual X-ray absorptiometry (model DCS-600R; Aloka, Tokyo, Japan), as reported previously. ${ }^{7)}$ The bone mineral content (BMC) of the femurs was closely correlated with the ash weight. ${ }^{7}$ The BMD was calculated by dividing the BMC of the measured area by the area.

Statistical Analysis — The data are expressed as the means \pm SEM. The significance of differences was analyzed using Student's $t$-test.

\section{RESULTS}

\section{LPS Induces COX-2 and mPGES-1 mRNAs in Mouse Osteoblasts to Produce PGE}

We performed RT-PCR to examine the effects of LPS on the mRNA expression of COX1, COX-2, cPGES, and mPGES-1 in mouse primary osteoblasts (Fig. 1A). LPS markedly induced the mRNA expression of COX-2 and mPGES- 1 in osteoblasts at $3 \mathrm{hr}$. The expression of COX-1 and cPGES mRNAs was detected in osteoblasts and its expression was not affected by LPS. The level of $\mathrm{PGE}_{2}$ in the conditioned medium of mouse osteoblasts cultured in medium containing $10 \%$ FCS was measured at $24 \mathrm{hr}$, and we detected a marked elevation of $\mathrm{PGE}_{2}$ in the conditioned medium of mouse osteoblasts treated with LPS (Fig. 1B).

\section{Effects of EP4 Antagonist on LPS-induced Os- teoclast Formation in Cocultures of Mouse Bone Marrow Cells and Osteoblasts}

We next examined the effects of LPS on osteoclast formation and $\mathrm{PGE}_{2}$ production in cocultures of mouse osteoblasts and bone marrow cells. Adding LPS to the cocultures markedly induced osteoclast formation and $\mathrm{PGE}_{2}$ production, both of which were completely suppressed by adding indomethacin, an inhibitor of PG synthesis (Fig. 2). When EP4 antagonist was added to the cocultures, LPS-induced osteoclast formation was completely suppressed, and the level of $\mathrm{PGE}_{2}$ in conditioned medium was not changed (Fig. 2). Therefore, the EP4 signal is essential for osteoclast formation induced by LPS in the coclutures.

EP4 Antagonist Recovers LPS-induced Bone Loss of Mouse Alveolar Bone in a Model for Periodontitis

Periodontitis is a typical inflammatory bone dis-

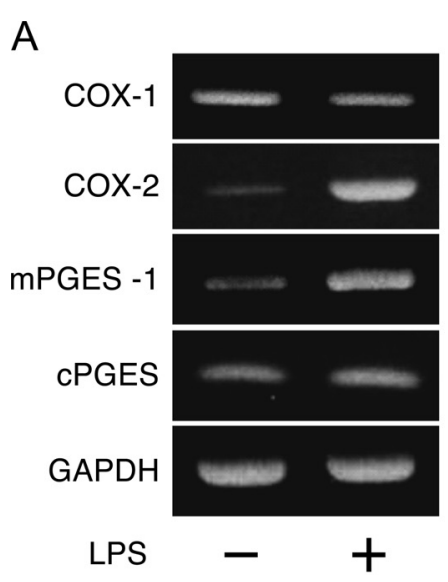

B

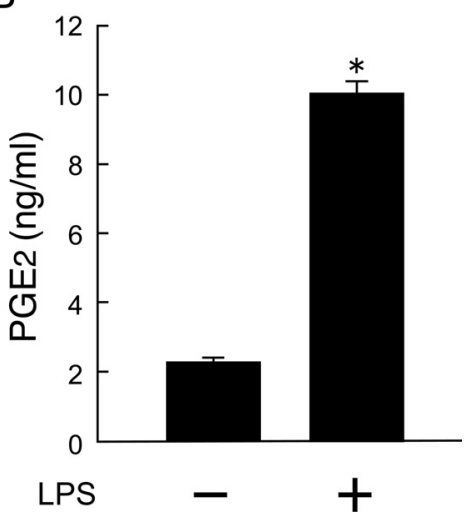

Fig. 1. Effects of LPS on $\mathrm{PGE}_{2}$ Production and mRNA Expression of COX-1, COX-2, mPGES-1, and cPGES in Mouse Osteoblasts

(A) Mouse primary osteoblasts were cultured for $3 \mathrm{hr}$ with or without $10 \mathrm{ng} / \mathrm{ml}$ of LPS, and total RNA was extracted. RT-PCR was performed using respective primers for mouse COX-1, COX-2, mPGES1, cPGES, and glyceraldehyde-3-phosphate dehydrogenase (GAPDH). (B) Osteoblasts were cultured for $24 \mathrm{hr}$ with or without $10 \mathrm{ng} / \mathrm{ml}$ of LPS, and the concentration of $\mathrm{PGE}_{2}$ in the culture medium was determined using EIA. Data are expressed as the means \pm SEM of 3 wells. Significantly different from the control without LPS, ${ }^{*} p<0.001$.

ease accompanying severe alveolar bone loss. To examine the mechanism of the pathogenesis of periodontitis, we recently developed a new experimental model for periodontitis. ${ }^{17)}$ When mandibular alveolar bone was collected from mice, and cultured with or without LPS to detect bone resorption, we could detect an increase in bone-resorbing activity by treatment with LPS in alveolar bone $e x$ vivo. EP4 antagonist, 0.01-1 $\mu \mathrm{M}$, dose-dependently suppressed the bone-resorbing activity induced by LPS in the organ culture of mouse mandibular alveolar bone (Fig. 3A). The level of $\mathrm{PGE}_{2}$ was elevated by the LPS treatment, and not influenced by EP4 antagonist (Fig. 3B). Indomethacin also suppressed LPS-induced $\mathrm{PGE}_{2}$ production and bone-resorbing activity in the cultures (data not shown). In in vivo experiments, LPS was injected into the gingiva in 

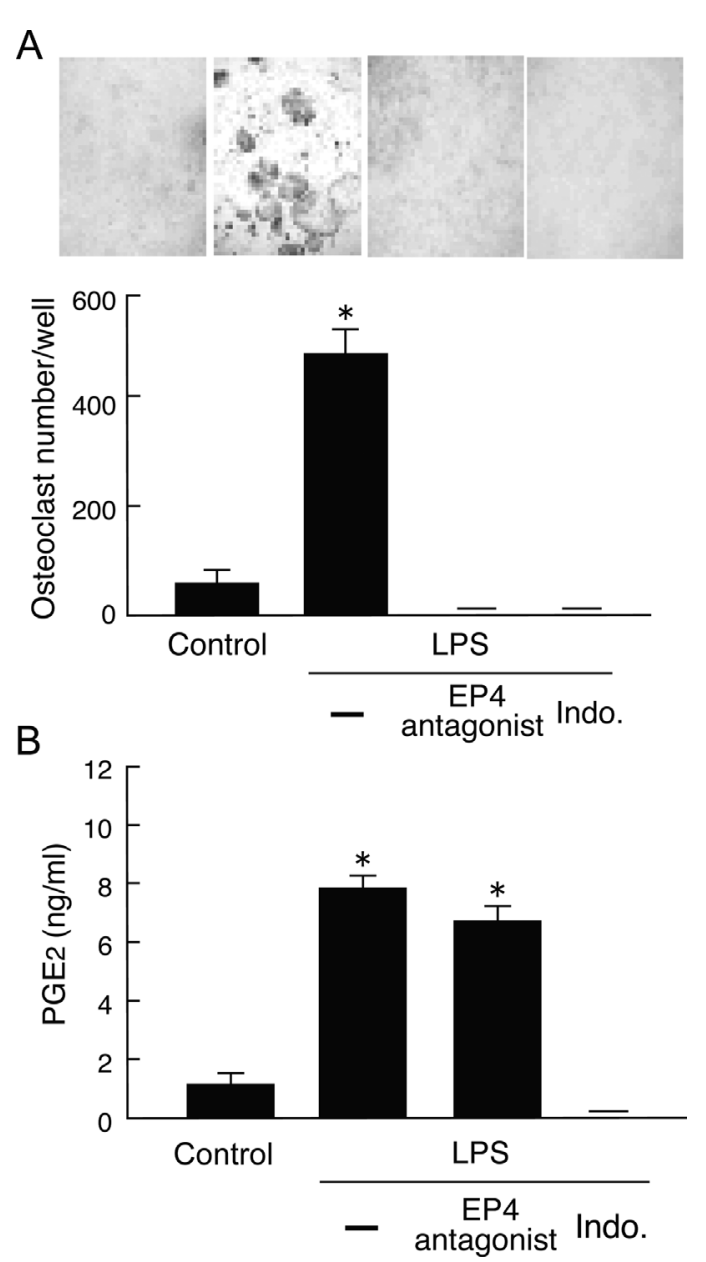

Fig. 2. EP4 Antagonist Suppresses Osteoclast Formation Induced by LPS in Cocultures of Mouse Bone Marrow Cells and Osteoblasts

Mouse bone marrow cells $\left(3 \times 10^{6}\right.$ cells $)$ and osteoblasts $\left(1 \times 10^{4}\right.$ cells) were cocultured for 7 days in the presence or absence of $1 \mathrm{ng} / \mathrm{ml}$ of LPS with or without $1 \mu \mathrm{M}$ EP4 antagonist and $1 \mu \mathrm{M}$ indomethacin. (A) Representative fields of TRAP staining are shown (upper panel). The number of osteoclasts was counted (lower panel). (B) Using the conditioned medium of the cocultures shown in panel A, the concentration of $\mathrm{PGE}_{2}$ was determined by EIA. Significantly different, ${ }^{*} p<0.001$ versus control. Data are expressed as the means \pm SEM of four wells.

the lower mandible, and alveolar bone was collected from mice on day 7 for the measurement of BMD by dual X-ray absorptiometry. LPS administration induced a significant decrease in BMD of mandibular alveolar bone (Fig. 4). Treatment with EP4 antagonist in the lower mandible recovered the bone loss induced by LPS in a dose-dependent manner (Fig. 4).

\section{DISCUSSION}

$\mathrm{PGE}_{2}$ production by osteoblasts is regulated in three metabolic steps: the release of arachi-
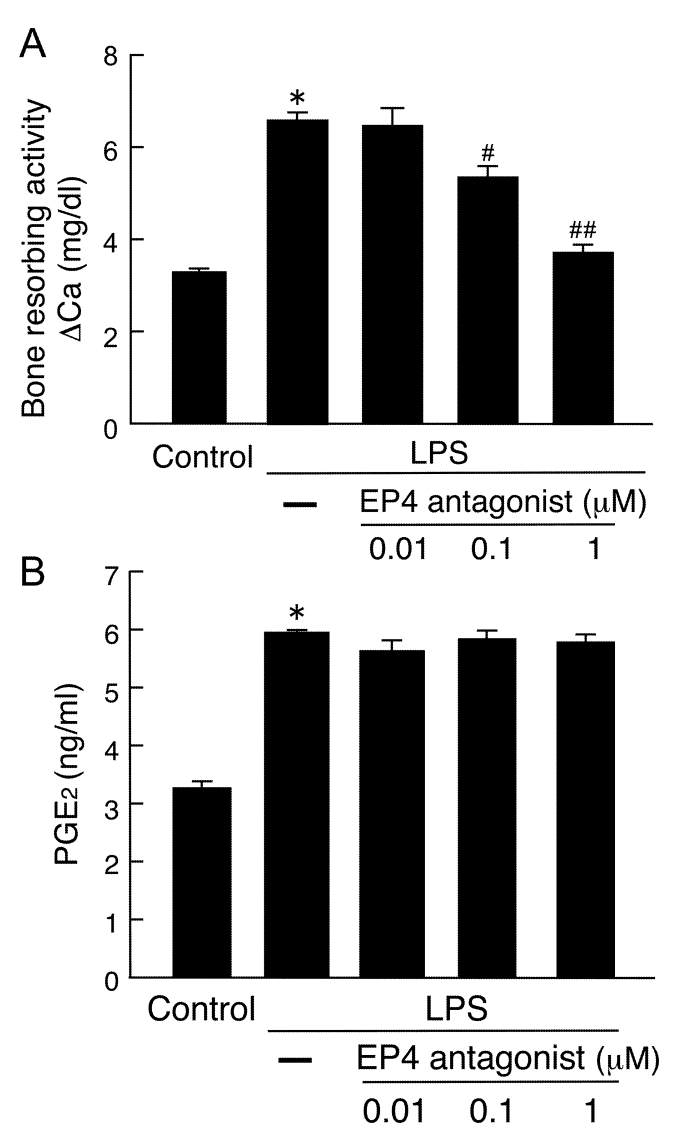

Fig. 3. EP4 Antagonist Suppresses Bone Resorption Induced by LPS in Organ Culture of Mouse Mandibular Alveolar Bone

Alveolar bone was collected from the mouse lower mandible, and cultured for 5 days with or without $1 \mathrm{mg} / \mathrm{ml}$ of LPS. EP4 antagonist, $0.01-1 \mu \mathrm{M}$, was added to the cultures in the presence of LPS. The boneresorbing activity was measured by medium calcium, as described in the Materials and Methods. (B) Using the conditioned medium of the organ cultures shown in panel $\mathrm{A}$, the concentration of $\mathrm{PGE}_{2}$ was determined by EIA. Significantly different from the control without LPS, ${ }^{*} p<0.001$ versus control, ${ }^{\#} p<0.05,{ }^{\# \#} p<0.001$ versus LPS. Data are expressed as the means \pm SEM of 3 cultures.

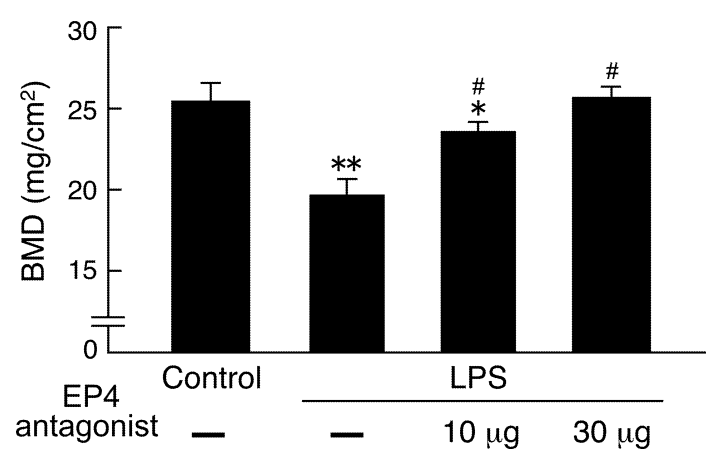

Fig. 4. EP4 Antagonist Recovers the LPS-induced Bone Loss of Mandibular Alveolar Bone In Vivo

LPS or PBS was injected into the gingiva in the lower mandible of mice, as described in the Materials and Methods, and the alveolar bone was collected from mice on day 7 for the measurement of BMD. Significantly different from control mice injected with PBS, ${ }^{*} p<0.05$, ${ }^{* *} p<0.001$, and ${ }^{\#} p<0.001$ versus LPS. Data are expressed as the means $\pm \mathrm{SEM}$ of four mice. 
donic acid from the membranous phospholipids by $\mathrm{CPLA}_{2} \alpha$, the conversion of arachidonic acid to $\mathrm{PGH}_{2}$ by $\mathrm{COX}-2$, and the synthesis of $\mathrm{PGE}_{2}$ by PGES. We have reported that $\mathrm{CPLA}_{2} \alpha$ expression in osteoblasts is indispensable to the arachidonic acid release for $\mathrm{PGE}_{2}$ synthesis and bone resorption, using PLA $_{2} \alpha$-deficient mice. ${ }^{7)} \mathrm{COX}-2$ is known to be induced by cytokines, such as IL-1 in osteoblasts. Recent studies have indicated that mPGES-1 is an inducible terminal enzyme for $\mathrm{PGE}_{2}$ biosynthesis, and its expression is linked to COX-2 induced by inflammatory cytokines in various cells. ${ }^{6)}$ In the present study, LPS markedly induced the mRNA expression of COX-2 and mPGES-1. Therefore, $\mathrm{PGE}_{2}$ production by osteoblasts may be regulated by the sequential pathway of $\mathrm{cPLA}_{2} \alpha$-COX-2-mPGES- 1 .

TLR4 has been reported to be the most essential LPS receptor for signal transduction. ${ }^{16)}$ To understand the pathway of LPS-induced $\mathrm{PGE}_{2}$ production in osteoblasts, we used $\mathrm{C} 3 \mathrm{H} / \mathrm{HeJ}$ mice with mutated Tlr4. Osteoblasts from $\mathrm{C} 3 \mathrm{H} / \mathrm{HeJ}$ mice did not respond to LPS, and $\mathrm{PGE}_{2}$ production was not altered. Also, LPS-induced bone loss in the femur was impaired in $\mathrm{C} 3 \mathrm{H} / \mathrm{HeJ}$ mice. ${ }^{17)}$ Thus, LPS binds to TLR4 on osteoblasts, and directly induces the expression of COX-2 and mPGES-1 for PGE $_{2}$ synthesis, leading to subsequent osteoclast formation.

It is well known that $\mathrm{PGE}_{2}$ acts on osteoblasts and induces the expression of RANKL to elicit osteoclastogenesis. We have reported that $\mathrm{PGE}_{2}$ could not induce bone resorption in organ cultures of mouse calvariae collected from EP4-null mice, and that EP4 agonist stimulates RANKL expression in osteoblasts and osteoclast formation in bone marrow cultures. ${ }^{13,14)}$ In the present study, we clearly showed the effects of EP4 antagonist on osteoclast formation induced by LPS in the cocultures of mouse bone marrow cells and osteoblasts. We also detected the suppressive effects of EP4 antagonist on bone resorbing activity in mouse calvarial cultures (data not shown). Therefore, EP4 antagonist may prevent bone loss associated with inflammation.

In the previous study, we demonstrated a new model for mouse periodontitis associated with a loss of alveolar bone, which is induced by LPS administration to an ex vivo organ culture and in vivo injection to alveolar bone. The model successfully showed bone loss induced by LPS. In the present study, EP4 antagonist suppressed the LPS-induced loss of alveolar bone in an organ culture of mouse alveolar bone, and in an in vivo model of periodonti- tis (Figs. 3 and 4). Salvi and Lang ${ }^{18)}$ have reviewed evidence from animal experiments and clinical trials documenting that selective and non-selective nonsteroidal anti-inflammatory drugs (NSAIDs) were responsible for the stabilization of periodontal conditions by reducing the rate of alveolar bone resorption. In our model, like EP4 antagonist, indomethacin also suppressed alveolar bone loss induced by LPS (data not shown). Therefore, the application of EP4 antagonist and/or NSAIDs may help to protect against the loss of alveolar bone in periodontal diseases.

In the present study, we clearly showed that EP4 antagonist suppressed LPS-induced osteoclast formation and alveolar bone resorption, and that EP4 antagonist prevented the destruction of mouse mandibular alveolar bone. The LPS-TLR4-COX-2mPGES-1-EP4 pathway may be critical for $\mathrm{PGE}_{2}$ production and alveolar bone loss in the pathogenesis of periodontitis, and EP4 antagonist may be a promising therapeutic application to inflammatory bone disease.

\section{REFERENCES}

1) Yasuda, H., Shima, N., Nakagawa, N., Yamaguchi, K., Kinosaki, M., Mochizuki, S., Tomoyasu, A., Yano, K., Goto, M., Murakami, A., Tsuda, E., Morinaga, T., Higashio, K., Udagawa, N., Takahashi, N. and Suda, T. (1998) Osteoclast differentiation factor is a ligand for osteoprotegerin/osteoclastogenesis-inhibitory factor and is identical to TRANCE/RANKL. Proc. Natl. Acad. Sci. U.S.A., 31, 3597-3602.

2) Lacey, D. L., Timms, E., Tan, H. L., Kelley, M. J., Dunstan, C. R., Burgess, T., Elliott, R., Colombero, A., Elliott, G., Scully, S., Hsu, H., Sullivan, J., Hawkins, N., Davy, E., Capparelli, C., Eli, A., Qian, Y. X., Kaufman, S., Sarosi, I., Shalhoub, V., Senaldi, G., Guo, J., Delaney, J. and Boyle, W. J. (1998) Osteoprotegerin ligand is a cytokine that regulates osteoclast differentiation and activation. Cell, 17, 165176.

3) Fuller, K., Wong, B., Fox, S., Choi, Y. and Chambers, T. J. (1998) TRANCE is necessary and sufficient for osteoblast-mediated activation of bone resorption in osteoclasts. J. Exp. Med., 188, 9971001.

4) Kudo, I., Murakami, M., Hara, S. and Inoue, K. (1993) Mammalian non-pancreatic phspholipase A2. Biochem. Biophys. Acta, 117, 217-231. 
5) Smith, W. L. and DeWitt, D. L. (1996) Prostaglandin endoperoxide $\mathrm{H}$ synthase-1 and -2. Adv. Immunol., 62, 167-215.

6) Murakami, M., Naraba, H., Tanioka, T., Semmyo, N., Nakatani, Y., Kojima, F., Ikeda, T., Fueki, M., Ueno, A., Oh, S. and Kudo, I. (2000) Regulation of prostaglandin E2 biosynthesis by inducible membrane-associated prostaglandin E2 synthase that acts in concert with cyclooxygenase-2. J. Biol. Chem., 275, 32783-32792.

7) Miyaura, C., Inada, M., Matsumoto, C., Ohshiba, T., Uozumi, N., Shimizu, T. and Ito, A. (2003) An essential role of cytosolic phospholipase A2 $\alpha$ in prostaglandin E2-mediated bone resorption associated with inflammation. J. Exp. Med., 197, 13031310.

8) Chen, Q. R., Miyaura, C., Higashi, S., Murakami, M., Kudo, I., Saito, S., Hiraide, T., Shibasaki, Y. and Suda, T. (1997) Activation of cytosolic phospholipase A2 by platelet-derived growth factor is essential for cyclooxygenase-2-dependent prostaglandin E2 synthesis in mouse osteoblasts cultured with interleukin-1. J. Biol. Chem., 272, 5952-5958.

9) Jakobsson, P. -J., Thoren, S., Morgenstern, R. and Samuelsson, B. (1999) Identification of human prostaglandin E synthase: A microsomal, glutathione-dependent, inducible enzyme, constituting a potential novel drug target. Proc. Natl. Acad. Sci. U.S.A., 96, 7220-7225.

10) Tanioka, T., Nakatani, Y., Semmyo, N., Murakami, M. and Kudo, I. (2000) Molecular identification of cytosolic prostaglandin E2 synthase that is functionally coupled with cyclooxygenase- 1 in immediate prostaglandin E2 biosynthesis. J. Biol. Chem., 275, 32775-32782.

11) Tanikawa, N., Ohmiya, Y., Ohkubo, H., Hashimoto, K., Kangawa, K., Kojima, M., Ito, S. and Watanabe,
K. (2002) Identification and characterization of a novel type of membrane-associated prostaglandin $\mathrm{E}$ synthase. Biochem. Biophys. Res. Commun., 291, 884-889.

12) Ushikubi, F., Hirata, M. and Narumiya, S. (1995) Molecular biology of prostanoid receptors; an overview. J. Lipid Mediat. Cell Signal., 12, 343359.

13) Suzawa, T., Miyaura, C., Inada, M., Maruyama, T., Sugimoto, Y., Ushikubi, F., Ichikawa, A., Narumiya, S. and Suda, T. (2000) The role of prostaglandin E receptor subtypes (EP1, EP2, EP3, and EP4) in bone resorption: An analysis using specific agonists for the respective EPs. Endocrinology, 141, 1554-1559.

14) Miyaura, C., Inada, M., Suzawa, T., Sugimoto, Y., Ushikubi, F., Ichikawa, A., Narumiya, S. and Suda, T. (2000) Impaired bone resorption to prostaglandin E2 in prostaglandin E receptor EP4-knockout mice. J. Biol. Chem., 275, 19819-19823.

15) Akira, S. and Takeda, K. (2004) Toll-like receptor signalling. Nat. Rev. Immunol., 4, 499-511.

16) Takeuchi, O., Hoshino, K., Kawai, T., Sanjo, H., Takada, H., Ogawa, T., Takeda, K. and Akira, S. (1999) Differential roles of TLR2 and TLR4 in recognition of gram-negative and gram-positive bacterial cell wall components. Immunity, 11, 443-451.

17) Inada, M., Matsumoto, C., Uematsu, S., Akira, S. and Miyaura, C. (2006) Membrane-bound PGE synthase-1-mediated $\mathrm{PGE}_{2}$ production by osteoblast plays a critical rule in LPS-induced bone loss associated with inflammation. J. Immunol., 177, 18791885.

18) Salvi, G. E. and Lang, N. P. (2005) The effects of non-steroidal anti-inflammatory drugs (selective and non-selective) on the treatment of periodontal diseases. Curr. Pharm. Des., 11, 1757-1769. 